\title{
An archipelago within an archipelago: A checklist of liverworts and hornworts of Kepulauan Sunda Kecil (Lesser Sunda Islands), Indonesia and Timor-Leste (East Timor)
}

\author{
Ainun Nadhifah', Lars Söderström², Anders Hagborg², \\ Eka Aditya Putri Iskandar', Ida Haerida ${ }^{4}$, Matt von Konrat ${ }^{3}$
}

I Cibodas Botanic Garden, Research Center for Plant Conservation and Botanic Gardens, Indonesian Institute of Sciences (LIPI), West Java, Indonesia 2 Norwegian University of Science and Technology, Trondheim, Norway 3 The Field Museum, Chicago, USA 4 Herbarium Bogoriense, Research Center for Biology, Indonesian Institute of Sciences (LIPI), West Java, Indonesia

Corresponding author: Ainun Nadhifah (ainun.nadhifah@lipi.go.id)

Academic editor: Matt Renner | Received 11 March 2021 | Accepted 7 June 2021 | Published 3 August 2021

Citation: Nadhifah A, Söderström L, Hagborg A, Iskandar EAP, Haerida I, Konrat M (2021) An archipelago within an archipelago: A checklist of liverworts and hornworts of Kepulauan Sunda Kecil (Lesser Sunda Islands), Indonesia and Timor-Leste (East Timor). PhytoKeys 180: 1-30. https://doi.org/10.3897/phytokeys.180.65836

\begin{abstract}
The first ever liverwort and hornwort checklist is provided for the Kepulauan Sunda Kecil (Lesser Sunda Islands) of Indonesia and Timor-Leste (East Timor). We report 129 accepted taxa, 12 doubtful taxa and three rejected taxa previously reported for the Lesser Sunda Islands. The list is based on over 130 literature references, including monographs, regional studies, and molecular investigations. It is clear that bryophytes from this region have been overlooked historically, and under collected, compared to seed plants, birds, and other organisms, forming a remarkable gap in the flora of Indonesia. Publications dealing with liverworts of Lesser Sunda Islands are few and scattered. We predict that further fieldwork, in addition to collections unveiled from regional herbaria, will uncover a number of new records that remain to be reported, especially considering that regionally widespread species have been recorded elsewhere.
\end{abstract}

\section{Keywords}

Checklist, hornworts, Indonesia, Kepulauan Nusa Tenggara, Kepulauan Sunda Kecil, Lesser Sunda Islands, liverworts, Timor-Leste (East Timor)

Copyright Ainun Nadhifah et al. This is an open access article distributed under the terms of the Creative Commons Attribution License (CC BY 4.0), which permits unrestricted use, distribution, and reproduction in any medium, provided the original author and source are credited. 


\section{Introduction}

The Lesser Sunda Islands (LSI), known in Indonesia as Kepulauan Sunda Kecil, are an area covering a longitudinal distance of some 600 kilometres in the southeastern portion of Indonesia, extending between Java in the west and New Guinea in the east (Fig. 1). Lesser Sunda Islands include a multitude of islands, the major ones of which are Flores, Sumba, Sumbawa, and Timor. The region overlaps with two different countries; Indonesia, which includes four different provinces, i.e., Bali, West Nusa Tenggara, East Nusa Tenggara (including Western part of Timor island), and part of Moluccas (van Steenis-Kruseman and van Steenis 1950; Monk et al. 1997; Jepson and Whittaker 2002) and Timor-Leste (East Timor), which includes the eastern part of Timor island (Kusuma 2017). The Lesser Sunda Islands occur as two geologically distinct island chains termed the Inner and Outer Banda Arcs (Audley-Charles 2011). This archipelago also occurs at the heart of the complex crossroads of two continents, Asia and Australia, and two oceans, the Indian and Pacific (Monk et al. 1997). The Lesser Sunda Islands may act as 'stepping stones' for animals and plants dispersing between the Greater Sunda Shelf, i.e., the Malay Peninsula, Borneo, Sumatra, Java, and Bali, and the Sahul Shelf, i.e., New Guinea, Australia, and their land-bridge islands (Reilly 2016). Bordered to the west by the Greater Sunda Shelf and to the east by the Sahul Shelf, the Lesser Sunda Islands can be considered oceanic islands in the sense that they have never been connected by land to continental Asia or continental Australo-Papua.

The island chain of LSI comprises $5.2 \%$ of endemic species based on families treated in Flora Malesiana (van Welzen et al. 2005). Interestingly, the Indonesian part contains the highest percentages of endemic plants of Indonesia, i.e., 55\% (Bappenas 2016). Some important and better-known endemic species in this ecoregion are the Komodo dragon (Varanus komodoensis), the largest lizard in the world and the iconic

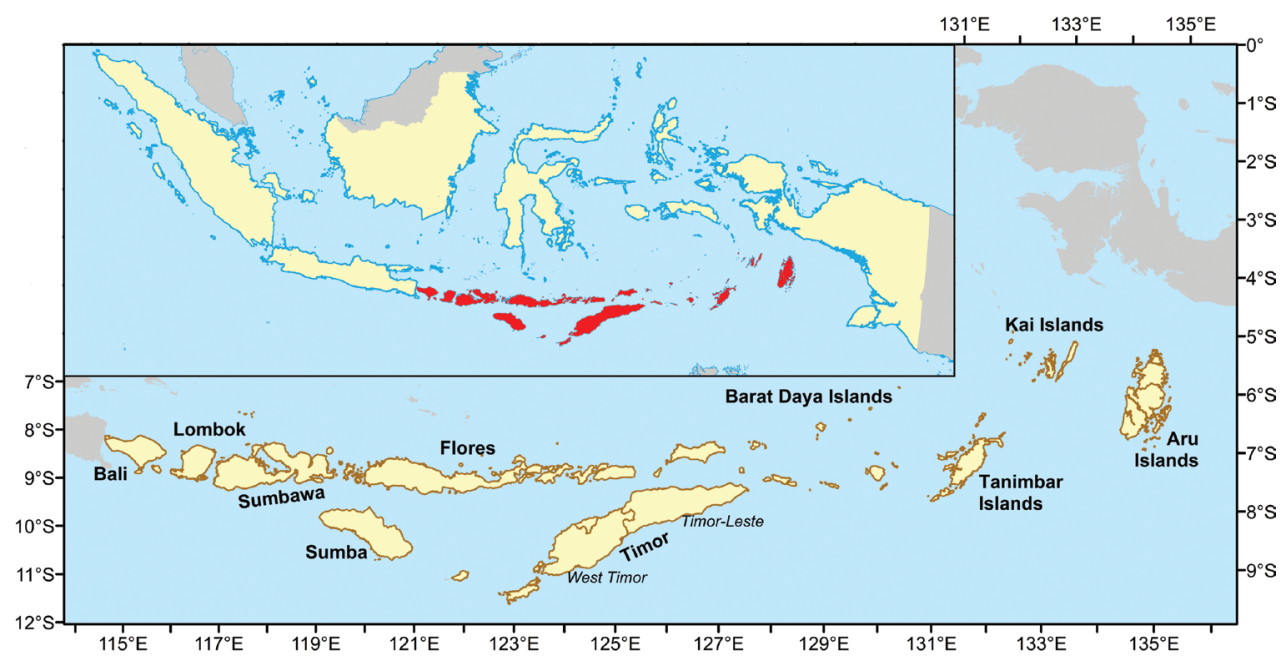

Figure I. Map of Lesser Sunda Islands with an inset of Indonesia. 
tree of East Nusa Tenggara Province, sandalwood tree (Santalum album L.), and Dracaena multiflora Warb. ex Sarasin (Monk et al. 1997).

The islands of LSI include seven ecoregions in two biomes, Tropical and Subtropical Moist Forests and Tropical and Subtropical Dry Forests (Fig. 2; Terrestrial Ecoregions of the World; Olson et al. 2001). The "Tropical and Subtropical Moist forest" includes two ecoregions on Bali, and two ecoregions on the eastern islands. The Eastern Java-Bali rainforest (https://www.worldwildlife.org/ecoregions/ im0113) and Eastern Java-Bali Montane Rain Forest (https://www.worldwildlife. org/ecoregions/im0112) are situated on the Sunda shelf and both are classified as endangered ecoregions since a lot of forest has been cleared. It forms transitional vegetation types relative to the drier areas of the central islands situated on the Sahul shelf.

The "Tropical and Subtropical Dry forests" consist of three ecoregions from Lombok in the west to Timor and Wetar in the east. All three ecoregions are critically endangered/endangered. The largest of the ecoregions is "Lesser Sunda Deciduous Forests" (https:/www.worldwildlife.org/ecoregions/aa0201) on Lombok, Sumbawa, Flores and west to Alor including smaller surrounding islands. It consists mainly of semi-evergreen dry forests. With an average annual rainfall of $1,349 \mathrm{~mm}$, this is the driest area but also the most seasonal in Indonesia (Touw 1992). The "Sumba Deciduous Forest" (https://www.worldwildlife.org/ecoregions/aa0203)" was also dominated by deciduous monsoon forest, but now much of it has been replaced by savanna and grasslands (Monk et al. 1997). The "Timor and Wetar Deciduous Forest" (https:// www.worldwildlife.org/ecoregions/aa0204) is also largely deforested and replaced by grasslands and savanna.

Further east, two more ecoregions of "Tropical and Subtropical Moist Deciduous Forests” occur. The "Banda Sea Islands Moist Deciduous Forest” (https://www.worldwildlife.org/ecoregions/aa0102) includes all islands west of Timor/Wetar, except Aru Island. It includes evergreen rain forest (Kepulauan Kai), semi-evergreen rain forest, moist deciduous forest, and dry deciduous forest (Monk et al. 1997). The forests are still largely intact, but the ecoregion is still classified as vulnerable. The "Vogelkop-Aru Lowland

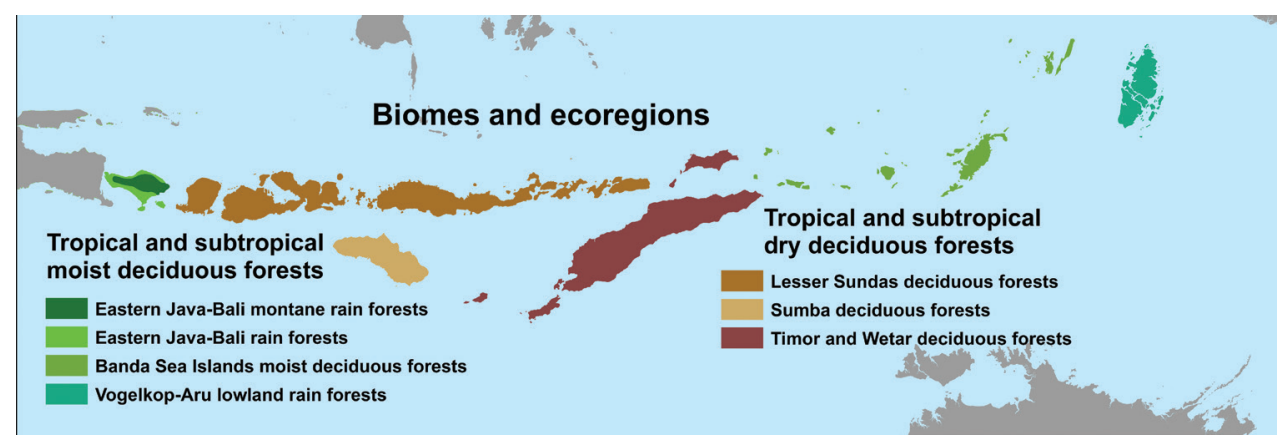

Figure 2. Seven ecoregions in two biomes of Lesser Sunda Islands following the Terrestrial Ecoregions of the World. 
Rain Forest" (https://www.worldwildlife.org/ecoregions/im0128) is mostly confined to New Guinea but occurs also on Aru. Large parts of the ecoregion are still intact.

Bryophytes, including mosses, liverworts and hornworts, are the second largest group of land plants after flowering plants and are pivotal in our understanding of early land plant evolution (e.g., Ligrone et al. 2012; Zhang et al. 2020). Bryophytes play a significant ecological role including $\mathrm{CO}_{2}$ exchange (De Lucia et al. 2003), plant succession (Cremer and Mount 1965), production and phytomass (Frahm 1990), nutrient cycling (Coxson 1992) and water retention (Pócs 1980; Gradstein et al. 2001). Retnowati et al. (2019) cited 849 species of liverworts, 28 species of hornworts and 1,884 species of mosses which are scattered in the major islands of Indonesia. As with many regions in the world, it is evident that the bryophyte, especially the liverwort flora, remains very poorly known in comparison to the vascular flora. Gradstein and Culmsee (2010) noted there are few studies from Southeast Asia investigating the diversity and ecology of tropical bryophytes.

It is clear that bryophytes from this region have been overlooked historically, and under-collected, compared to seed plants, birds, and other organisms, forming a remarkable gap in the flora of Indonesia and/or Timor-Leste. Publications dealing with liverworts of LSI also are few and scattered. The first apparent report of liverworts from LSI was by Sande Lacoste (1856) in the mid- $19^{\text {th }}$ century. It was not until the late $19^{\text {th }}$ century and early $20^{\text {th }}$ century that further influential works appeared, including those by Schiffner $(1898,1900,1955)$, Stephani $(1886,1899,1907,1908$, 1909, 1911, 1917, 1924), Verdoorn (1930, 1934a, 1934b, 1935, 1937), and others. Among those islands, Bali is the most explored island (Hegewald and van Zanten 1986; Eggers et al. 1998; Schäfer-Verwimp 2006, 2009; Haerida et al. 2010; Alam 2012; Heinrichs et al. 2012; Girmansyah et al. 2013) with 101 species accepted here. Söderström and Séneca (2008) reported only 61 number of liverworts for Lesser Sunda Islands and considered that this low number of species was the effect of the under-explored areas. More recently, little botanical work has been done in the area; the area has occasionally been visited by students and researchers from nearby institutions, but publications are still lacking. This checklist will complement the survey of mosses of Lesser Sunda Islands by Touw (1992) who enumerated 367 species for the area, and complement other checklists of liverworts from Indonesia, including Java (Söderström et al. 2010), Bali (Haerida 2015, 2017) as well as Sumba (Haerida et al. 2020).

We here present the first-ever checklist of liverworts and hornworts for the Lesser Sunda Islands to serve as the baseline information in our study of the liverworts diversity of this archipelago. Furthermore, this checklist can serve to promote and encourage bryological research in the region. The significance of checklists is summarized by Söderström et al. (2008), including outlining the utility of checklists as powerful and important tools, and their applicability to taxonomy, systematics, and conservation.

As with many other regions in the world, given the relatively poor focus on liverworts in LSI historically, we predict that a vast number of new records are yet to 


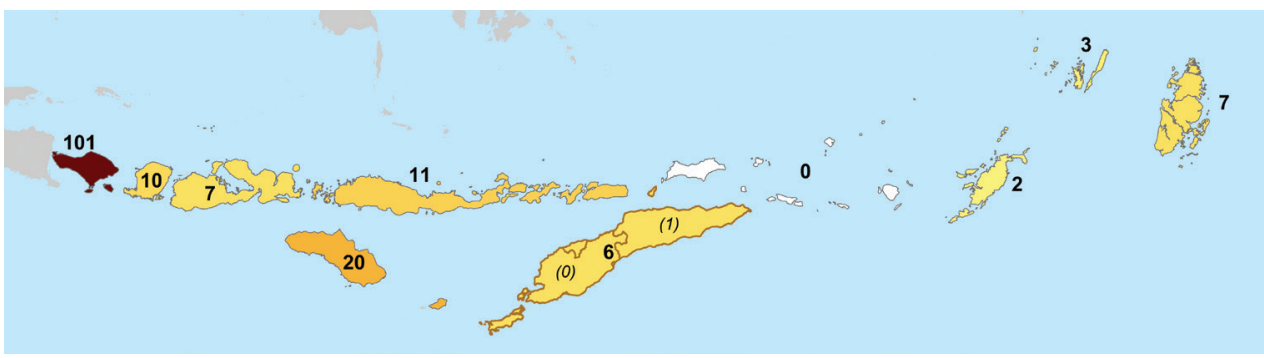

Figure 3. Number of known species from the individual islands. The darker the color, the greater the number of species.

be reported for the area. In this checklist we report 129 taxa, with another 12 taxa questioned and three rejected. The number of known species from individual islands varies from 101 (Bali) to 0 (Barat Daya Islands), but only Bali has more than 20 known species (Fig. 3).

\section{Materials and methods}

Nomenclature and taxonomy follows the world checklist of hornworts and liverworts (Söderström et al. 2016) with a few updates from recent taxonomic literature. Sources include over 130 publications found through the work of Early Land Plants Today (ELPT) database of liverwort taxonomy and distribution, and with some consultation with taxonomic experts. The checklist follows a similar format of previous liverwort and hornwort checklists by the authors, e.g., Java (Söderström et al. 2010). The checklist distinguishes between records that are based on specimens seen by the author(s) (reference in bold) and second-hand reports, e.g., citations of earlier publications (reference in normal type). All names used for the references are given after the taxon name with spelling variants/errors within quotation marks. Taxa are arranged in alphabetical order. Significantly, each accepted taxon is qualified using a four level ranking system that indicates our level of knowledge about a taxon. The coding convention is outlined in detail by von Konrat et al. (2010). Briefly, ? = Problem with the taxon name; ${ }^{*}=$ Serious doubts about the value of the taxon; ${ }^{* *}=$ Probably a good taxon (default value) ${ }^{* * *}=$ Accepted, a good taxon as currently understood.

This checklist covers the Lesser Sunda Islands based on reports from the literature that have been subdivided into 10 geographical units that correspond to geopolitical units. These include from west to east (Fig. 1) Bali, Lombok, Sumbawa, Sumba, Flores, Timor (separated in the Indonesian West Timor and the independent Timor-Leste), Barat Daya Islands, Tanimbar Islands, Kai Islands and Aru Islands. 


\section{Types from Lesser Sunda Islands}

Recently, there has been some debate whether the type information by Bonner (1962, $1963,1965)$, as well as the other volumes of his "Index Hepaticarum", can be accepted as lectotypes. Renner (2021) argued in favour for the volumes of Bonner (1962, 1963, 1965), including recommendations to improve typification practice, and Engel and Merrill (2019) argued that Bonner's herbarium designations do not represent lectotypifications. Here we accept the typifications made by Bonner as they pertain to the taxa treated here, but we also provide alternative typifications if ruled against. In the latter cases, we instead "validate" Bonner's typifications, yet causing no nomenclatural changes whichever view is taken.

It is not always clear if more than one collection exists for a possible type. McNeill (2014) recommends that in such a case a lectotype should be selected from known available material, but with a statement that it may be the only material, in which case a lectotypification would be superfluous. For such cases, we here follow McNeill's "best practice" advice using the suggested phrase "lectotype here designated, if not a holotype".

Anastrophyllum integerrimum Steph., Sp. Hepat. (Stephani) 6: 107, 1917 (Stephani 1917). Originally described from "Java (Koorders leg), Lombock. (Elbert legit.)". Lectotype (Bonner 1962): Java, 5000 pd., Koorders s.n. ex hb. Schinz, Zürich, G-00067196 (http://www.ville-ge.ch/musinfo/bd/cjb/chg/adetail.php?id=118174\&lang=en). Specimen also annotated as "holotype" by J. Váňa in 1974. Note: If Bonner's typification is rejected, we here designate the specimen as a new lectotype. The species was synonymized with Gottschelia schizopleura by Grolle (1968).

Chiloscyphus communis Steph., Bull. Herb. Boissier (sér. 2) 7 (10): 839 [=Sp. Hepat. (Stephani) 3: 211], 1907 (Stephani 1907). Originally described from "Java, Sumatra, Celebes, Nova Guinea, Timor, Samoa, Andaman Insulae, Queensland, Assam, Sikkim, Japan, valde communis.". Lectotype (Bonner 1963): Andamans, Port Blair, VIII.1890, E.H. Man, ex hb. Levier, G-00115054 (http://www.ville-ge.ch/ musinfo/bd/cjb/chg/adetail.php?id=165692\&lang=en). Specimen annotated as isotype by H. Inoue (undated). There are many specimens in G that Stephani may have seen, but apparently only this specimen corresponds to Bonner's typification. The species was synonymized with Chiloscyphus bescherellei (=Heteroscyphus coalitus) by Hattori (1966).

Drepanolejeunea moluccensis Herzog, Ann. Bryol. 7: 88, 1934 (Herzog 1934). Originally described from "Molukken: Batjan, G. Sibella (Herb. H. B. Bog. n. 4103, leg. Roepke); Bali: Bratansee (Renner n. 331 o u. đ̊)". Lectotype (here designated): Molukken, Batjan, G. Sibela, 1600-2000 m, W.K.J. Roepke s.n., JE-04002975 (https://herbarium.univie.ac.at/database/detail.php?ID=120304). Herzog noted "typus" on the specimen from Batjan while he noted "cotypus" on the specimen from Bali. The Bali material is issued in the exsiccatae "Hepaticae Selectae et Criticae (ed. Fr. Verdoorn) Ser. VIII (1965) 365" as "materia originalis" and should be present in several herbaria.

Fimbraria latifrons Steph., Sp. Hepat. (Stephani) 6: 15, 1917 (Stephani 1917). Originally described from “Lombock. (Sunda Archipelagus.) (Elbert legit.)". Lectotype 
(Long 2006: 227): [Indonesia] Lombok, Rindjani-Vulkangebirge, NNO Seite, Andjar Fluss, oberhalb; Monsun Hochwald, 1400-1530 m, humus Sand, 19.5.1909, J. Elbert 1388 (G-15225). Isolectotype: FH). [=G-00113144, http://www.ville-ge.ch/ musinfo/bd/cjb/chg/adetail.php?id=144318\&lang=en]. The species was synonymized with Reboulia hemisphaerica by Long (2006).

Frullania longispica Steph., Sp. Hepat. (Stephani) 4: 454, 1911 (Stephani 1911). Originally described from "Insula Timor". Type (Bonner 1965): Timor, 1100 m, IX 1897, Francis Newton s.n., G. However, there are two specimens in G with those collection data, G barcode 00069224 and 00265585 . The former is annotated by Verdoorn in 1928 as "typica, .... species auton. non est!" (http://www.ville-ge.ch/musinfo/ $\mathrm{bd} / \mathrm{cjb} / \mathrm{chg} /$ adetail.php?id=114964\&lang=en), the latter does not have any annotation. Verdoorn never published any typification of Frullania longispica but G00069224 is apparently the base for the synonymization with Frullania squarrosa in Verdoorn (1930) and is here designated as lectotype.

Lepidozia newtonii Steph., Sp. Hepat. (Stephani) 3: 623, 1909 (Stephani 1909). Originally described from "Insula Timor (Newton)". Lectotype (here designated, if not holotype): Timor, $1100 \mathrm{~m}$, Sept 1897, Francis Newton, Steph. herb. no. 126, G barcode 00069698 (http://www.ville-ge.ch/musinfo/bd/cjb/chg/adetail. php?id=135054\&lang=en). There seems to be only one specimen in $G$ that may be designated type, but we have not searched other herbaria and thus do not know if there are any possible type material elsewhere.

Madotheca elbertii Steph., Sp. Hepat. (Stephani) 6: 520, 1924 (Stephani 1924). Originally described from "India orientalis: Lombock (Elbert legit)". Lectotype (Hattori 1969): Lombock, leg. leg. Dr. Elbert 2016, type of Madotheca elbertii, in herb. G (G barcode no. 00043932, http://www.ville-ge.ch/musinfo/bd/cjb/chg/adetail.php?id=130660\&lang=en). The species was synonymized with Porella acutifolia var. lancifolia (= var. acutifolia) by Hattori (1976).

Mastigobryum sumbavense Steph., Hedwigia 25 (6): 236, 1886 (Stephani 1886). Originally described from "Insula Sumbawa, ad 4000', Zollinger No. 3400b (Herb. Gottsche)”. Lectotype (Mizutani 1967, 'type'): Insula Sumbava, Montis Batu, Alt. 4000 ft., Lante ad arbores, Zollinger 3400b, Steph. herb. no. 10769, G barcode 00066907. If Mizutani's type specification is rejected we here designate the mentioned specimen as new lectotype (if it is not a holotype).

\section{List of accepted species and infraspecific taxa}

\section{Anthocerotophyta}

\section{Folioceros}

*** Folioceros fuciformis (Mont.) D.C.Bharadwaj BALI: Schiffner 1955 as Aspiromitus falsinervius. 


\section{Marchantiophyta}

\section{Acanthocoleus}

*** Acanthocoleus javanicus (Steph.) Kruijt. BALI: Wilson et al. 2007; Heinrichs et al. 2012; Dong et al. 2013; Haerida 2017.

\section{Acrolejeunea}

*** Acrolejeunea aulacophora (Mont.) Steph. Lesser Sunda Is.: BALI: Hegewald and van Zanten 1986; Haerida 2017.

*** Acrolejeunea fertilis (Reinw., Blume et Nees) Schiffn. BaLI: Gradstein 1975; Wilson et al. 2004; Forrest et al. 2006; Wilson et al. 2007; Heinrichs et al. 2012; Czumay et al. 2013; Dong et al. 2013; Heinrichs et al. 2014a; Heinrichs et al. 2014b; Schäfer-Verwimp et al. 2014; Bechteler et al. 2016a; Bechteler et al. 2016b; Haerida 2017. Tanimbar Islands: Gradstein 1975. TIMOR: Gradstein 1975.

\section{Aneura}

*** Aneura maxima (Schiffn.) Steph. BaLI: Haerida 2017.

*** Aneura pinguis (L.) Dumort. BALI: Haerida et al. 2015; Haerida 2017.

\section{Asterella}

\footnotetext{
*** Asterella blumeana (Nees) Kachroo Loмвок: Long 2006.

*** Asterella vulcanica (Schiffn.) Kachroo et Bapna BALI: Haerida et al. 2015; Haerida 2017.
}

\section{Bazzania}

*** Bazzania longicaulis (Sande Lac.) Schiffn. Sumba: Haerida et al. 2020.

* ${ }^{1}$ Bazzania sumbavensis (Gottsche ex Steph.) Steph. Sumbawa: Lectotype of Mastigobryum sumbavense, Stephani 1886 as Mastigobryum sumbavense, Schiffner 1898; Stephani 1908 as Mastigobryum sumbavense, Bonner 1963; Grolle 1966; Mizutani 1967; Tixier 1974; Kamimura 1975; Miller et al. 1983; Miller et al. 1983 as Mastigobryum sumbavense, Geissler and Bischler 1985 as Mastigobryum sumbavense, Long and Grolle 1990; Sharma and Srivastava 1993; Bapna and Kachroo 2000.

1 Bazzania sumbavensis is possibly conspecific with Bazzania japonica (Söderström et al. 2010). 


\section{Cheilolejeunea}

** Cheilolejeunea ceylanica (Gottsche) R.M.Schust. et Kachroo Sumba: Haerida et al. 2020.

*** Cheilolejeunea trapezia (Nees) Kachroo et R.M.Schust. BaLI: Haerida 2017. Sumba: Haerida et al. 2020.

\section{Chiastocaulon}

*** Chiastocaulon dendroides (Nees) Carl. BaLI: So 2001 as Plagiochila dendroides.

*** Chiastocaulon oppositum (Reinw., Blume et Nees) S.D.F.Patzak, M.A.M.Renner, Schäf.-Verw. et Heinrichs. Lesser Sunda Is.: Pócs et al. 2011 as Plagiochilion oppositum. BALI: Hegewald and van Zanten 1986 as Plagiochilion oppositum, Groth and Heinrichs 2005 as Plagiochilion oppositum, Haerida et al. 2015 as Plagiochilion oppositum, Patzak et al. 2016; Renner et al. 2016; Haerida 2017; Renner et al. 2017. Sumbawa: Sande Lacoste $\mathbf{1 8 6 4}$ as Plagiochila opposita, Schiffner 1898 as Plagiochila opposita, Miller et al. 1983 as Plagiochilion oppositum.

\section{Cololejeunea}

*** Cololejeunea angustiflora (Steph.) Mizut. BaLI: Haerida 2017.

*** Cololejeunea appressa (A.Evans) Benedix. BaLI: Haerida 2017.

** Cololejeunea gottschei (Steph.) Pandé, K.P.Srivast. et Ahmad Balı: Haerida 2017.

*** Cololejeunea macounii (Spruce) A.Evans BaLI: Haerida 2017.

*** Cololejeunea obliqua (Nees et Mont.) Schiffn. BALI: Benedix 1953 as Cololejeunea nymannii, Tixier 1962 as Cololejeunea nymannii, Tixier 1973 as Cololejeunea nymannii, Miller et al. 1983 as Cololejeunea nymannii.

*** Cololejeunea ocelloides (Horik.) Mizut. BaLI: Tixier 1962 as Cololejeunea leonidens var. saccata.

** Cololejeunea subfloccosa Mizut. BALI: Haerida 2017.

** Cololejeunea triapiculata (Herzog) Tixier BaLI: Haerida 2017.

*** Cololejeunea trichomanis (Gottsche) Besch. BaLI: Haerida 2017 as Cololejeunea goebelii.

\section{Colura}

** ${ }^{2}$ Colura leratii (Steph.) Steph. BaLI: Eggers et al. 1998; Haerida 2017. Flores: Jovet-Ast 1967 as Colura apiculata, Eggers et al. 1998.

2 Colura leratii was omitted in Söderström et al. (2016) as the basionym was erroneously considered invalid. 


\section{Diplasiolejeunea}

*** Diplasiolejeunea cavifolia Steph. Balr: Schäfer-Verwimp 2006³ ; Haerida 2017; Siregar et al. 2020.

\section{Drepanolejeunea}

*** Drepanolejeunea levicornua Steph. Sumba: Haerida et al. 2020.

** Drepanolejeunea moluccensis Herzog. BaLI: SyntyPe, Herzog 1934; Verdoorn 1935; Tixier 1979.

*** Drepanolejeunea pentadactyla (Mont.) Steph. BaLr: Haerida 2017.

\section{Dumortiera}

*** Dumortiera hirsuta (Sw.) Nees Ball: Haerida et al. 2015; Haerida 2017.

\section{Fossombronia}

*** Fossombronia himalayensis Kashyap. BALI: Krayesky et al. 2005.

\section{Frullania}

** Frullania apiculata (Reinw., Blume et Nees) Nees Sumba: Haerida et al. 2020.

*** Frullania ericoides (Nees) Mont. BaLI: Hegewald and van Zanten 1986; Haerida 2017. Lомвок: Verdoorn 1934b as Frullania squarrosa. Timor: TyPE of Frullania longispica, Stephani 1911 as Frullania longispica, Bonner 1965 as Frullania longispica.

*** Frullania gaudichaudii (Nees et Mont.) Nees et Mont. BaLI: Haerida 2015; Haerida 2017.

*** Frullania gracilis (Reinw., Blume et Nees) Nees BALI: Haerida 2015; Haerida 2017; Rosyanti et al. 2018.

** Frullania intermedia (Reinw., Blume et Nees) Nees subsp. intermedia. ARU Islands: Hattori 1980.

*** Frullania junghuhniana Gottsche. BaLI: Haerida 2015; Haerida 2017.

*** Frullania meyeniana Lindenb. BaLI: Haerida 2015; Haerida 2017; Rosyanti et al. 2018.

3 The collecting data for the report of Diplasiolejeunea cavifolia in Schäfer-Verwimp (2006) is "Nordbali, Distr. Tabanan, Bedugul, Aufstieg im Nebelwald zum Gunung Catur am Bratansee, epiphyll an Pandanus sp.; 1260 m, 30. Mai 1995, leg. Schäfer-Verwimp \& Verwimp, det. Schäfer-Verwimp (Hb Schäfer-Verwimp No. 16750)" (A. Schäfer-Verwimp in litt. 2021). 
${ }^{* * *}{ }^{4}$ Frullania moniliata (Reinw., Blume et Nees) Mont. BatI: Haerida 2015; Haerida 2017. Sumba: Haerida et al. 2020. Sumbawa: Sande Lacoste 1856; Schiffner 1898; Verdoorn 1930 as Frullania moniliata subsp. breviramea, Hattori 1975 as Frullania tamarisci var. breviramea.

*** Frullania nodulosa (Reinw., Blume et Nees) Nees ARU Islands: Mitten 1885 as Frullania secundiflora, Schiffner $\mathbf{1 8 9 8}$ as Frullania secundiflora. Flores: Hattori 1975. KaI IsLands: Mitten 1885 as Frullania secundiflora, Schiffner 1898 as Frullania secundiflora, Miller et al. 1983. Sumba: Verdoorn 1930; Haerida et al. 2020. Sumbawa 5 : Hattori 1951; Swanson and Miller 1969; Miller et al. 1983. Tanimbar IsLANDS: Hattori 1980 as Frullania nodulosa var. nodulosa. TIMOR: Hattori 1980 as Frullania nodulosa var. nodulosa.

*** Frullania ornithocephala (Reinw., Blume et Nees) Nees Bald: Haerida 2015; Haerida 2017. Lомвок: Verdoorn 1934b.

*** Frullania rio-janeirensis (Raddi) Ångstr. BaLI: Hegewald and van Zanten 1986; Enroth 1991 as Frullania africana, Haerida 2017.

*** Frullania serrata Gottsche Lомвок: Verdoorn 1934b.

*** Frullania ternatensis Gottsche BaLI: Haerida 2015; Haerida 2017.

** — var. non-appendiculata S.Hatt. BALI: Hegewald and van Zanten 1986.

${ }^{*}$ Frullania tricarinata Sande Lac. BALI: Hegewald and van Zanten 1986 as Frullania 'tricapinata', Haerida 2017; Winter and Schäfer-Verwimp 2020.

*** Frullania trichodes Mitt. KaI IsLands: Verdoorn 1937 as Frullania tenuicaulis.

\section{Gottschelia}

*** Gottschelia schizopleura (Spruce) Grolle Lesser Sunda Is.: Váňa and Piippo 1989b; Váňa 1991b. Lомвок: SyNTYPE of Anastrophyllum integerrimum, Stephani 1917 as Anastrophyllum integerrimum, Bonner 1962 as Anastrophyllum integerrimum, Grolle 1968; Miller et al. 1983 as Anastrophyllum integerrimum.

\section{Herbertus}

** Herbertus ceylanicus (Steph.) Abeyw. FLores: Juslén 2006.

*** Herbertus dicranus (Gottsche, Lindenb. et Nees) Trevis. Bali: Juslén 2006; Váňa et al. 2014.

** Herbertus longispinus J.B.Jack et Steph. Flores: Juslén 2006.

** Herbertus ramosus (Steph.) H.A.Mill. Balr: Hegewald and van Zanten 1986; Haerida 2017.

4 Frullania moniliata is a species complex (Vilnet et al. 2014) in sect. Thyopsiella.

5 We do not know of any first hand report of Frullania nodulosa from Sumbawa.

6 Frullania tricarinata is possibly conspecific with Frullania hypoleuca (Söderström et al. 2010). 
*** Herbertus sendtneri (Nees) Lindb. Lesser Sunda Is.: Juslén 2006 as Herbertus armitanus.

\section{Heteroscyphus}

*** Heteroscyphus argutus (Reinw., Blume et Nees) Schiffn. ARu Islands: Mitten 1885 as Chiloscyphus argutus, Schiffner $\mathbf{1 8 9 8}$ as Chiloscyphus argutus. Balr: Haerida et al. 2015; Haerida 2017.

*** Heteroscyphus aselliformis (Reinw., Blume et Nees) Schiffn. Balr: Haerida 2017. Sumbawa: Zollinger 1855 as Chiloscyphus aselliformis, Sande Lacoste 1856 as Chiloscyphus aselliformis, Schiffner $\mathbf{1 8 9 8}$ as Chiloscyphus aselliformis, Schiffner 1900 as Chiloscyphus aselliformis, Miller et al. 1983; Piippo 1985; Piippo 1989b; Yamada and Hayashi 2003.

*** Heteroscyphus coalitus (Hook.) Schiffn. BaLI: Hegewald and van Zanten 1986 as Chiloscyphus coalitus, Srivastava and Srivastava 2002; Haerida et al. 2015; Haerida 2017. Sumba: Haerida et al. 2020. Timor: SyntyPE of Chiloscyphus communis, Stephani 1907 as Chiloscyphus communis, Hattori 1951 as Heteroscyphus communis, Miller 1968 as Chiloscyphus communis, Swanson and Miller 1969 as Chiloscyphus communis, Piippo 1993. Timor-Leste: Piippo 1985; Piippo 1989b.

*** Heteroscyphus splendens (Lehm. et Lindenb.) Grolle Sumba ${ }^{7}$ : Miller et al. 1983 as Heteroscyphus decurrens. Sumbawa: Zollinger 1855 as Chiloscyphus decurrens, Sande Lacoste 1856 as Chiloscyphus decurrens, Schiffner 1898 as Chiloscyphus decurrens, Pócs 1971 as Heteroscyphus decurrens.

Jackiella

*** Jackiella javanica Schiffn. BaLI: Haerida 2017.

\section{Lejeunea}

*** Lejeunea alata Gottsche Sumba: Haerida et al. 2020.

*** Lejeunea apiculata Sande Lac. BaLI: Haerida 2017.

*** Lejeunea mimula Hürl. BaLI: Wilson et al. 2004; Gradstein et al. 2006; Wilson et al. 2007; Heinrichs et al. 2012; Czumay et al. 2013; Dong et al. 2013; Heinrichs et al. 2014a; Heinrichs et al. 2014b; Haerida 2017.

\section{Lepidozia}

* Lepidozia newtonii Steph. Timor: Type, Stephani 1909.

7 We do not know of any first hand report of Heteroscyphus splendens from Sumba. It may be an error for Sumbawa. 


\section{Leptolejeunea}

*** Leptolejeunea elliptica (Lehm. et Lindenb.) Besch. Sumba: Haerida et al. 2020.

*** Leptolejeunea epiphylla (Mitt.) Steph. BaLI: Haerida 2017.

** Leptolejeunea foliicola Steph. BaLI: Eggers et al. 1998; Bechteler et al. 2016c; Haerida 2017; Shu and Zhu 2019.

* Leptolejeunea massartiana Schiffn. ex Herzog BaLI: Eggers et al. 1998; Haerida 2017.

*** Leptolejeunea subacuta Steph. ex A.Evans BalI: Haerida 2017.

\section{Lopholejeunea}

*** Lopholejeunea eulopha (Taylor) Schiffn. BALI: Zhu and Gradstein 2005; Haerida et al. 2010; Haerida 2017; Siregar et al. 2020.

*** Lopholejeunea horticola Schiffn. BaLr: Zhu and Gradstein 2005; Haerida et al. 2010; Siregar et al. 2014; Siregar 2015; Pócs and Chantanaorrapint 2016; Haerida 2017.

*** Lopholejeunea nigricans (Lindenb.) Schiffn. Bali: Haerida 2017. Sumba: Haerida et al. 2020.

*** Lopholejeunea recurvata Mizut. BaLI: Zhu and Gradstein 2005; Haerida et al. 2010; Haerida 2017.

*** Lopholejeunea subfusca (Nees) Schiffn. Lesser Sunda Is.: Pócs et al. 1967. Aru IsLaNDS: Mitten 1885 as Lejeunea subfusca, Schiffner 1898 as Lopholejeunea sagrana var. subfusca. Bali: Zhu and Gradstein 2005; Haerida 2009; Haerida et al. 2010; Siregar et al. 2014; Siregar 2015; Haerida 2017; Rosyanti et al. 2018; Siregar et al. 2020.

*** Lopholejeunea zollingeri (Steph.) Schiffn. BaLI: Haerida 2017.

\section{Marchantia}

*** Marchantia acaulis Steph. BaLI: Haerida et al. 2015; Haerida 2017.

*** Marchantia emarginata Reinw., Blume et Nees Balr: Siregar et al. 2013; Haerida et al. 2015; Siregar 2015; Haerida 2017; Ginting and Batubara 2019.

*** — subsp. emarginata. Ball: Bischler-Causse 1989; Bischler and Piippo 1991. FLores: Bischler-Causse 1989; Bischler and Piippo 1991.

*** Marchantia geminata Reinw., Blume et Nees BaLI: Haerida et al. 2015; Haerida 2017. FLORES: Bischler-Causse 1989.

*** Marchantia treubii Schiffn. LesSer Sunda Is.: Siregar et al. 2013; Siregar 2015. BALI: Haerida 2017. Flores: Bischler-Causse 1989. LоMBOK: Bischler-Causse 1989. Timor: Stephani 1899; Bischler-Causse 1989.

\section{Metalejeunea}

*** Metalejeunea cucullata (Reinw., Blume et Nees) Grolle BaLI: Bechteler et al. 2016b. 


\section{Metzgeria}

*** Metzgeria ciliata Raddi BaLI: Haerida 2017.

*** Metzgeria consanguinea Schiffn. Sumba: Haerida et al. 2020.

* Metzgeria foliicola Schiffn. Flores: So 2003b.

*** Metzgeria lindbergii Schiffn. Bali: Haerida 2017. Flores: So 2003 b.

\section{Pallavicinia}

*** Pallavicinia lyellii (Hook.) Gray Balı: Haerida 2017.

\section{Plagiochila}

*** Plagiochila bantamensis (Reinw., Blume et Nees) Mont. Bald: So 2001.

*** Plagiochila frondescens (Nees) Lindenb. BaLI: Inoue 1984; Piippo 1989a; Patzak et al. 2016; Renner et al. 2017.

*** Plagiochila javanica (Sw.) Nees et Mont. BaLr: Inoue 1984; Hegewald and van Zanten 1986; Piippo 1989a; Haerida 2017.

*** Plagiochila junghuhniana Sande Lac. BaLI: So 2001.

${ }^{*}{ }^{8}$ Plagiochila kubliana Sande Lac. BaLI: Inoue 1984.

** Plagiochila massalongoana Schiffn. BALI: Inoue 1984.

*** Plagiochila obtusa Lindenb. Bali: So 2001.

*** Plagiochila parvifolia Lindenb. BaLI: So 2001.

** Plagiochila propinqua Sande Lac. Balr: Inoue 1984; Inoue 1989; Piippo 1989a; Piippo and Tan 1992; Grolle and So 1999b.

*** Plagiochila renitens (Nees) Lindenb. BaLI: Inoue 1984; Inoue 1989; Piippo 1989a.

*** Plagiochila salacensis Gottsche BALI: Carl 1931 as Plagiochila jackii, Hegewald and van Zanten 1986; Grolle and So 1999a; So 2001; Siregar 2015; Haerida 2017; Siregar et al. 2018.

*** Plagiochila sciophila Nees Balr: Inoue 1984; Inoue 1989; Piippo 1989a; Enroth 1991; Siregar 2015; Siregar et al. 2018.

** Plagiochila semidecurrens (Lehm. et Lindenb.) Lindenb. BaLI: So 2001.

** Plagiochila spathulifolia Mitt. BaLI: Inoue 1984; Inoue 1989.

*** Plagiochila teysmannii Sande Lac. BaLI: Hegewald and van Zanten 1986; So and Grolle 1999; Haerida 2017. Sumba: Haerida et al. 2020.

\section{Pleurozia}

*** Pleurozia gigantea (F.Weber) Lindb. FLores: Thiers 1993.

8 Plagiochila kubliana is possibly conspecific with Plagiochila sciophila (Söderström et al. 2010). 


\section{Porella}

** Porella acutifolia (Lehm. et Lindenb.) Trevis. Balr: Hegewald and van Zanten 1986; Haerida 2017. Lомвок: Miller et al. 1983.

** - var. acutifolia. Lомвок: LестотүPE of Madotheca elbertii, Stephani 1924 as Madotheca elbertii, Hattori 1969 as Porella acutifolia var. elbertii.

\section{Ptychanthus}

*** Ptychanthus striatus (Lehm. et Lindenb.) Nees Bali: Haerida 2017. LomboK: Verdoorn 1934a; Miller et al. 1983. Sumba: Haerida et al. 2020.

\section{Radula}

** Radula acuminata Steph. BALI: Haerida 2017.

*** Radula campanigera Mont. BALI: Haerida 2017.

** Radula javanica Gottsche Balr: Haerida 2017. Sumba: Haerida et al. 2020.

* Radula multiflora Gottsche ex Schiffn. ARu Islands: Schiffner 1898. BalI: Hegewald and van Zanten 1986; Haerida 2017.

* Radula pinnulata Mitt. ARU IsLands: Mitten 1885; Schiffner 1898.

*** Radula ventricosa Steph. BaLI: Haerida 2017.

\section{Reboulia}

*** Reboulia hemisphaerica (L.) Raddi BaLI: Haerida et al. 2015; Haerida 2017. Lомвок: LеCтотуPE of Fimbraria latifrons, Stephani 1917 as 'Fimbriaria' latifrons. Bonner 1965 as 'Fimbriaria' latifrons, Long 2006.

\section{Riccardia}

** Riccardia crenulata Schiffn. BaLI: Schiffner 1955 as Riccardia tenuicostata. Sumba: Haerida et al. 2020 as Aneura crenulata.

\section{Riccia}

*** Riccia billardierei Mont. et Nees BaLI: Jovet-Ast 2000; Jovet-Ast 2003.

*** Riccia cruciata Kashyap. Balı: Jovet-Ast 2003.

*** Riccia discolor Lehm. et Lindenb. BaLr: Jovet-Ast 2003.

*** Riccia junghuhniana Nees et Lindenb. BalI: Jovet-Ast 2003; Haerida 2017.

*** Riccia mangalorica Ahmad ex Jovet-Ast BALI: Jovet-Ast 2003 as Riccia mangalorica.

\section{Scapania}

*** Scapania javanica Gottsche BALI: Blockeel et al. 2009; Haerida 2017. 


\section{Schiffneriolejeunea}

*** Schiffneriolejeunea tumida (Nees) Gradst. BALI: Wilson et al. 2004.

*** — var. haskarliana (Gottsche) Gradst. et Terken. BALI: Wilson et al. 2007.

\section{Schistochila}

*** Schistochila aligera (Nees et Blume) J.B.Jack et Steph. Balr: So 2003a. Flores: So 2003a.

*** Schistochila blumei (Nees) Trevis. BaLI: So 2003a.

\section{Solenostoma}

*** Solenostoma tetragonum (Lindenb.) R.M.Schust. ex Váňa et D.G.Long BALI: Váňa 1972, 1973, 1975, 1991a all as Jungermannia tetragona, Miller et al. 1983 as Jungermannia tetragona, Váňa and Piippo 1989a as Jungermannia tetragona, Bapna and Kachroo 2000 as Jungermannia tetragona, Srivastava and Sharma 2000 as Jungermannia tetragona, Easa 2003 as Jungermannia tetragona.

*** Solenostoma truncatum (Nees) R.M.Schust. ex Váňa et D.G.Long BaLI: Váňa and Piippo 1989a as Jungermannia truncata, Váňa 1991a as Jungermannia truncata, Bapna and Kachroo 2000 as Jungermannia truncata.

\section{Spruceanthus}

Spruceanthus polymorphus (Sande Lac.) Verd. ARU IsLands: Mitten 1885 as Phragmicoma polymorpha, Schiffner $\mathbf{1 8 9 8}$ as Thysananthus polymorphus. Sumba: Haerida et al. 2020.

*** Spruceanthus semirepandus (Nees) Verd. LomвoK: Verdoorn 1934a; Jovet-Ast and Schmid 1958; Kitagawa 1981.

\section{Thysananthus}

*** Thysananthus humilis (Gottsche) Sukkharak et Gradst. BaLI: Sukkharak and Gradstein 2014 as Mastigolejeunea humilis. Sumba: Haerida et al. 2020 as Mastigolejeunea humilis.

*** Thysananthus ligulatus (Lehm. et Lindenb.) Sukkharak et Gradst. Sumba: Haerida et al. 2020 as Mastigolejeunea ligulata.

*** Thysananthus spathulistipus (Reinw., Blume et Nees) Lindenb. Balr: Wilson et al. 2007; Haerida et al. 2010; Sukkharak 2011; Heinrichs et al. 2012; Czumay et al. 2013; Dong et al. 2013; Heinrichs et al. 2014a; Heinrichs et al. 2014b; Schäfer-Verwimp et al. 2014; Siregar et al. 2014; Siregar 2015; Sukkharak 2015; Bechteler et al. 2016a; Bechteler et al. 2016b; Haerida 2017; Siregar et al. 2017; Siregar et al. 2020. KaI IsLands: Verdoorn 1937. Sumbawa: 
Zollinger 1855; Schiffner 1898; Verdoorn 1934a; Swanson and Miller 1969; Miller et al. 1983; Haerida et al. 2010; Siregar et al. 2014; Siregar 2015; Siregar et al. 2017.

*** Thysananthus virens Ångstr. BALI: Sukkharak and Gradstein 2014b as Mastigolejeunea virens, Siregar et al. 2020 as Mastigolejeunea virens.

\section{Wiesnerella}

*** Wiesnerella denudata (Mitt.) Steph. BaLI: Haerida 2017.

\section{Taxa of unclear affinity}

A couple of taxa are published from Lesser Sunda Islands as varieties of species synonymized under other names, without transferring or synonymizing the variety. We have not been able to trace any specimen that they may be based on and, thus, not been able to refer them to any valid taxon.

\section{Chiloscyphus}

? Chiloscyphus zollingeri Gottsche var. subintegerrimus Schiffn. BaLI: Schiffner 1955. Note: Chiloscyphus zollingeri Gottsche is now Heteroscyphus zollingeri but we are not sure if var. subintegerrimus also belongs to that species and is worth recognizing.

\section{Riccardia}

? Riccardia platyclada Schiffn. var. leiomitra Schiffn. BaLI: Schiffner 1955. Note: Riccardia platyclada Schiffn. is a synonym of $R$. graeffei but it is unclear where var. leiomitra from Java belongs (Söderström et al. 2010).

Taxa reported but doubtfully occurring in Lesser Sunda Islands

\section{Marchantiophyta}

\section{Bazzania}

** Bazzania ceylanica (Mitt.) Steph. Lesser Sunda Is.: Miller et al. 1983. Note: The report by Miller et al. is unclear and it may be that they meant some of the Greater Sunda Islands. It is widespread in SE Asia, so its presence is not unlikely.

*** Bazzania erosa (Reinw., Blume et Nees) Trevis. LesSER Sunda Is.: Miller et al. 1983. Note: The report by Miller et al. is unclear and it may be that they meant some of the Greater Sunda Islands. It is widespread in SE Asia so its presence is not unlikely.

*** Bazzania tridens (Reinw., Blume et Nees) Trevis. Lesser Sunda Is.: Miller et al. 1983. Sumbawa: Pócs 1971. Note: We are not aware of any first hand report from 
Lesser Sunda Islands but it is common in SE Asia so its presence on some of the islands is not unlikely.

\section{Ceratolejeunea}

*** Ceratolejeunea cf. papuliflora Steph. Sumba: Haerida et al. 2020. Note: The species was only reported with doubt. It is otherwise not reported outside Africa, but it occurs on the Western Indian Ocean Islands and may perhaps also occur in SE Asia.

\section{Cololejeunea}

*** Cololejeunea cf. lanciloba Steph. BaLI: Haerida 2017. Note: The species was only reported with doubt. However, it is widespread in SE Asia and its occurrence in the area is not unlikely.

** Cololejeunea cf. serrulata Steph. Balr: Haerida 2017. Note: The species was only reported with doubt. It occurs on other Islands in SE Asia so its presence on the Lesser Sunda Islands is not unlikely.

\section{Colura}

*** Colura ari (Steph.) Steph. Lesser Sunda Is.: Miller et al. 1983. Note: The report by Miller et al. is unclear and it may be that they meant some of the Greater Sunda Islands. It is widespread in SE Asia so its presence is not unlikely.

** Colura imperfecta Steph. Lesser Sunda Is.: Miller et al. 1983. Note: The report by Miller et al. is unclear and it may be that they meant some of the Greater Sunda Islands. It is widespread in SE Asia so its presence is not unlikely.

\section{Conoscyphus}

*** Conoscyphus trapezioides (Sande Lac.) Schiffn. LesSER Sunda Is.: Miller et al. 1983 as Chiloscyphus trapezioides. Note: The report by Miller et al. is unclear and it may be that they meant some of the Greater Sunda Islands. It is widespread in SE Asia so its presence is not unlikely.

\section{Drepanolejeunea}

*** Drepanolejeunea ternatensis (Gottsche) Schiffn. LesSER Sunda Is.: Miller et al. 1983. Note: The report by Miller et al. is unclear and it may be that they meant some of the Greater Sunda Islands. It is widespread in SE Asia so its presence is not unlikely.

\section{Radula}

*** Radula complanata (L.) Dumort. BaLI: Haerida 2017. Note: A mainly boreal species that have its closest known occurrences in Himalaya. The report was 
erroneously published without a 'cf' as the identification was only preliminary (I. Haerida).

\section{Targionia}

Targionia hypophylla L. BALI: Haerida 2017. Note: The species was only reported with doubt. It is widespread but as the relation to other taxa remains unclear, its distribution is also unclear.

Taxa reported but rejected from Lesser Sunda Islands

\section{Marchantiophyta}

\section{Ceratolejeunea}

*** Ceratolejeunea ceratantha (Nees et Mont.) Schiffn. Sumbawa: Sande Lacoste 1864 as Lejeunea ceratantha, Schiffner 1898. Note: This is a Neotropical taxon and the old reports from Asia must be rejected.

\section{Frullania}

* Frullania ludoviciae Steph. Sumbawa: Miller et al. 1983 (with a '?'). Note: Hattori (1986) is rejecting earlier records outside New Caledonia as based on erroneous synonymization of F. tenuirostris.

\section{Thysananthus}

*** Thysananthus auriculatus (Wilson et Hook.) Sukkharak et Gradst. BaLI: Wilson et al. 2004 as Mastigolejeunea auriculata, Wilson et al. 2007 as Mastigolejeunea auriculata, Ye and Zhu 2018. NoтE: Sukkharak and Gradstein (2014) rejects all report of this American-African taxon from SE Asia as Thysananthus humilis.

\section{Synonyms}

Anastrophyllum integerrimum Steph. = Gottschelia schizopleura Aneura crenulata (Schiffn.) Steph. E Riccardia crenulata Aspiromitus falsinervius (Lindenb. ex Meissner) Steph. = Folioceros fuciformis Chiloscyphus argutus (Reinw., Blume et Nees) Nees $\equiv$ Heteroscyphus argutus Chiloscyphus aselliformis (Reinw., Blume et Nees) Nees $\equiv$ Heteroscyphus aselliformis Chiloscyphus coalitus (Hook.) Nees $\equiv$ Heteroscyphus coalitus

Chiloscyphus communis Steph. = Heteroscyphus coalitus

Chiloscyphus decurrens (Reinw., Blume et Nees) Nees = Heteroscyphus splendens

Chiloscyphus trapezioides Sande Lac. 三 Conoscyphus trapezioides

Cololejeunea goebelii (Gottsche ex Schiffn.) Schiffn. = Cololejeunea trichomanis 
Cololejeunea leonidens var. saccata Benedix = Cololejeunea ocelloides

Cololejeunea nymannii (Steph.) Benedix = Cololejeunea obliqua

Colura apiculata Steph. $=$ Colura leratii

Fimbraria latifrons Steph. $=$ Reboulia hemisphaerica

Frullania africana Steph. $=$ Frullania rio-janeirensis

Frullania longispica Steph. $=$ Frullania ericoides

Frullania moniliata subsp. breviramea (Steph.) Verd. = Frullania moniliata

Frullania secundiflora Mont. = Frullania nodulosa

Frullania squarrosa (Mont.) Nees = Frullania ericoides

Frullania tamarisci var. breviramea (Steph.) S.Hatt. = Frullania moniliata

Frullania tenuicaulis Mitt. $=$ Frullania trichodes

Herbertus armitanus (Steph.) H.A.Mill. = Herbertus sendtneri

Heteroscyphus communis (Steph.) Schiffn. = Heteroscyphus coalitus

Heteroscyphus decurrens (Nees) Schiffn. = Heteroscyphus splendens

Jungermannia tetragona Lindenb. 三 Solenostoma tetragonum

Jungermannia truncata Nees $\equiv$ Solenostoma truncatum

Lejeunea ceratantha Nees et Mont. $\equiv$ Ceratolejeunea ceratantha

Lejeunea subfusca (Nees) Nees et Mont. 三Lopholejeunea subfusca

Lopholejeunea sagrana var. $\beta$ subfusca (Nees) Schiffn. $\equiv$ Lopholejeunea subfusca

Madotheca elbertii Steph. = Porella acutifolia var. acutifolia

Mastigobryum sumbavense Gottsche ex Steph. 三 Bazzania sumbavensis

Mastigolejeunea auriculata (Wilson et Hook.) Steph. 三 Thysananthus auriculatus

Mastigolejeunea humilis (Gottsche) Schiffn. $\equiv$ Thysananthus humilis

Mastigolejeunea ligulata (Lehm. et Lindenb.) Schiffn. 三 Thysananthus ligulatus

Mastigolejeunea virens (Ångstr.) Steph. $\equiv$ Thysananthus virens

Phragmicoma polymorpha Sande Lac. $\equiv$ Spruceanthus polymorphus

Plagiochila dendroides (Nees) Lindenb. 三 Chiastocaulon dendroides

Plagiochila jackii Schiffn. nom. illeg. = Plagiochila salacensis

Plagiochila opposita (Reinw., Blume et Nees) Lindenb. 三 Chiastocaulon oppositum

Plagiochilion oppositum (Reinw., Blume et Nees) S.Hatt. 三 Chiastocaulon oppositum

Porella acutifolia var. elbertii (Steph.) S.Hatt. = Porella acutifolia var. acutifolia

Riccardia tenuicostata Schiffn. nom. illeg. = Riccardia crenulata

Riccardia tenuicostata Schiffn. = Riccardia inconspicua

Riccia mangalorica Ahmad nom. inval. $\equiv$ Riccia mangalorica

Thysananthus polymorphus (Sande Lac.) Schiffn. 三 Spruceanthus polymorphus

\section{Acknowledgements}

We would like to thank Research and Innovation in Science and Technology Project (RISET-Pro) - Ministry of Research and Technology/National Research and Innovation Agency of Indonesia for granting the scholarship to the first author, Cibodas Botanic Garden, Research Center for Plant Conservation and Botanic Gardens - LIPI for support, and Mr. Lukman Budiman from PDDI - LIPI for his kindly assistance in the BO library. 
The Biodiversity Heritage Library is acknowledged for the facility they provide that has greatly accelerated our efforts. The generous support by the National Science Foundation (Award No.'s 1145898, 1146168, 1458300, 1541506, 2001509 and 0531730) is gratefully acknowledged. We also recognize the support of the Museum Collection Spending Fund, administered by Field Museum, as well as curatorial support provided by Y. Rodriguez, L. Kawasaki, and A. Balla (Field Museum). Alfons Schäfer-Verwimp kindly provided us with specimen data from his unpublished Diplasiolejeunea cavifolia. We are very grateful for the helpful suggestions and comments made by the editor and reviewers: Matt A. Renner, S.R. Gradstein, Li Zhang, and an anonymous reviewer.

\section{References}

Alam A (2012) Liverwort Flora of Parson's valley Nilgiri hills (Western Ghats), South India. Archive for Bryology 119: 1-20.

Audley-Charles MG (2011) Tectonic post-collision processes in Timor. Geological Society of London, Special Publications 355(1): 241-266. https://doi.org/10.1144/SP355.12

Bapna KR, Kachroo P (2000) Hepaticology in India - I. Himanshu Publications, Udaipur, 439 pp. Bappenas [Kementerian Perencanaan Pembangunan Nasional/Badan Perencanaan Pembangunan Nasional/Indonesian Ministry of National Development Planning/ National Development Planning Board] (2016) Indonesian Biodiversity Strategy and Action Plan (IBSAP) 2015-2020. Jakarta.

Bechteler J, Lee GE, Schäfer-Verwimp A, Renner MAM, Peralta DF, Heinrichs J (2016a) Towards a monophyletic classification of Lejeuneaceae V: The systematic position of Pictolejeunea. Phytotaxa 280(3): 259-270. https://doi.org/10.11646/phytotaxa.280.3.4

Bechteler J, Lee GE, Schäfer-Verwimp A, Pócs T, Peralta DF, Renner MAM, Schneider H, Heinrichs J (2016b) Towards a monophyletic classification of Lejeuneaceae IV: Reinstatement of Allorgella, transfer of Microlejeunea aphanella. Plant Systematics and Evolution 302(2): 187-201. https://doi.org/10.1007/s00606-015-1252-8

Bechteler J, Schäfer-Verwimp A, Lee GE, Feldberg K, Pérez-Escobar OA, Pócs T, Peralta DF, Renner MAM, Heinrichs J (2016c) Geographical structure, narrow species ranges, and Cenozoic diversification in a pantropical clade of epiphyllous leafy liverworts. Ecology and Evolution 7(2): 638-653. https://doi.org/10.1002/ece3.2656

Benedix EH (1953) Indomalayische Cololejeuneen. Feddes Repertorium Specierum Novarum Regni Vegetabilis (Beiheft 134): 1-88.

Bischler H, Piippo S (1991) Bryophyte flora of the Huon Peninsula, Papua New Guinea. L. Marchantia (Marchantiaceae, Hepaticae). Annales Botanici Fennici 28(4): 277-301.

Bischler-Causse H (1989) Marchantia L. The Asiatic and Oceanic taxa. Bryophytorum Bibliotheca 38: 1-317.

Blockeel TL, Bastos CJP, Bednarek-Ochyra H, Ochyra R, Dulin MV, Fovet L, García C, Hedenäs L, Hugonnot V, Kirmaci M, Koponen T, Lebouvier M, Martins A, Müller F, Sabovljević M, Lakušić D, Schäfer-Verwimp A, Sérgio C, Surina B, Yayintaş ÖT (2009) New national and regional bryophyte records, 22. Journal of Bryology 31(3): 202-210. https://doi.org/10.1179/037366809X12469790518367 
Bonner CEB (1962) Index hepaticarum. Pars II. Achiton to Balantiopsis. J. Cramer, Weinheim, $320 \mathrm{pp}$.

Bonner CEB (1963) Index hepaticarum. Pars III. Barbilophozia to Ceranthus. J. Cramer, Weinheim, $636 \mathrm{pp}$.

Bonner CEB (1965) Index Hepaticarum. Pars V. Delavayella to Geothallus. J. Cramer, Weinheim, 480 pp.

Carl H (1931) Die Arttypen und die systematische Gliederung der Gattung Plagiochila Dum. Annales Bryologici (suppl. 2): 1-170.

Coxson D (1992) Nutrient release from epiphytic bryophytes in tropical montane rain forest. Canadian Journal of Botany 69(10): 2122-2129. https://doi.org/10.1139/b91-266

Cremer KW, Mount AB (1965) Early stages of plant succession following the complete felling and burning of Eucalyptus regnans forest in the Florentine Valley, Tasmania. Australian Journal of Botany 13: 303-322. https://doi.org/10.1071/BT9650303

Czumay A, Dong S, Scheben A, Schäfer-Verwimp A, Feldberg K, Heinrichs J (2013) Transfer of Lejeunea huctumalcensis to Physantholejeunea (Lejeuneaceae, Porellales). Australian Systematic Botany 26(5): 386-392. https://doi.org/10.1071/SB13039

De Lucia EH, Turnbull MH, Walcroft AS, Griffen KL, Tissue DT, Glenny D, McSeventy TM, Whitehead D (2003) The contribution of bryophytes to the carbon exchange for a temperate rainforest. Global Change Biology 9(8): 1158-1170. https://doi.org/10.1046/j.13652486.2003.00650.x

Dong S, Schäfer-Verwimp A, Pócs T, Feldberg K, Czumaj A, Schmidt AR, Schneider H, Heinrichs J (2013) Size doesn't matter - recircumscription of Microlejeunea (Lejeuneaceae, Porellales) based on molecular and morphological evidence. Phytotaxa 85(2): 41-55. https://doi.org/10.11646/phytotaxa.85.2.2

Easa PS (2003) Biodiversity documentation for Kerala, part 4 Bryophytes. Kerala Forest Research Handbook 17: 1-47.

Eggers J, Frahm J-P, Pursell RA (1998) New bryophyte taxon records for tropical countries II. Tropical Bryology 14: 81-84. https://doi.org/10.11646/bde.14.1.12

Engel JJ, Smith Merrill GL (2019) Bonner's “Types” are not lectotypes. Nova Hedwigia 109(1/2): 63-64. https://doi.org/10.1127/nova_hedwigia/2019/0545

Enroth J (1991) On the phytogeography of Western Melanesian Hepaticae. A literature review. The Journal of the Hattori Botanical Laboratory 70: 1-42.

Forrest LL, Davis EC, Long DG, Crandall-Stotler BJ, Clark A, Hollingsworth ML (2006) Unravelling the evolutionary history of the liverworts (Marchantiophyta): Multiple taxa, genomes and analyses. The Bryologist 109(3): 303-334. https://doi.org/10.1639/00072745(2006)109[303:UTEHOT]2.0.CO;2

Frahm J-P (1990) Bryophyte phytomass in tropical ecosystems. Botanical Journal of the Linnean Society 104(1-3): 23-33. https://doi.org/10.1111/j.1095-8339.1990.tb02209.x

Geissler P, Bischler H (1985) Index hepaticarum vol. 10. Lembidium to Mytilopsis. J. Cramer, Berlin, 352 pp.

Ginting N, Batubara MS (2019) The diversity of liverworts in the Dolok Sordang Sub-District of Sipirok, South Tapanuli, Indonesia. Budapest international research in exact sciences 1(2): 55-64. https://doi.org/10.33258/birex.v1i2.226 
Girmansyah D, Santika Y, Retnowati A, Wardani W, Haerida I, Widjaja EA, van Balgooy MMJ [Eds] (2013) Flora of Bali: an Annotated Checklist. Bogor: Herbarium Bogoriense, Botany Division, Research Center for Biology-LIPI \& Yayasan Pustaka Obor Indonesia.

Gradstein SR (1975) A taxonomic monograph of the genus Acrolejeunea (Hepaticae), with an arrangement of the genera of Ptychanthoideae. Bryophytorum Bibliotheca 4: 1-162.

Gradstein R, Culmsee H (2010) Bryophyte diversity on tree trunks in montane forests of Central Sulawesi Indonesia. Tropical Bryology 31: 95-105. https://doi.org/10.11646/bde.31.1.16

Gradstein SR, Churchill SP, Salazar-Allen N (2001) Guide to the Bryophytes of Tropical America. The New York Botanical Garden Press, New York.

Gradstein SR, Wilson R, Ilkiu-Borges AL, Heinrichs J (2006) Phylogenetic relationships and neotenic evolution of Metzgeriopsis (Lejeuneaceae) based on chloroplast DNA. Botanical Journal of the Linnean Society 151(3): 293-308. https://doi.org/10.1111/j.10958339.2006.00531.x

Grolle R (1966) Die Lebermoose Nepals. Khumbu Himal. Ergebnisse des Forschungsunternehmens Nepal Himalaya 1(4): 262-298. https://doi.org/10.1007/978-3-642-92914-4_5

Grolle R (1968) Gottschelia - eine neue Jungermanniales-Gattung der Paläotropis. The Journal of the Hattori Botanical Laboratory 31: 13-19.

Grolle R, So ML (1999a) Studies of Plagiochila sect. Subtropicae in Asia. The Bryologist 102(1): 67-75. https://doi.org/10.2307/3244462

Grolle R, So ML (1999b) Studies on Plagiochila sect. Tayloriae in Asia. The Bryologist 102(2): 287-293. https://doi.org/10.2307/3244366

Groth H, Heinrichs J (2005) Maximum likelihood analyses of chloroplast gene rbcL sequences indicate relationships of Syzygiella (Jungermanniopsida) with Lophoziaceae rather than Plagiochilaceae. Cryptogamie. Bryologie 26(1): 49-57.

Haerida I (2009) Keanekaragaman suku Lejeuneaceae (Hepaticae, Lumut Hati) di daerah sekitar PPKAB (Pusat Pendidikan dan Konservasi Alam Bodogol) Taman Nasional Gunung Gede-Pangrango, Jawa Barat [Diversity of Lejeuneaceae from surrounding area of Bodogol Education Center and Conservation, Gunung Gede-Pangrango National Park]. Berita biologi 9(4): 683-691.

Haerida I (2015) The genus Frullania (Hepaticae: Frullaniaceae) in Bali. Floribunda 5(2): $71-$ 73. https://doi.org/10.32556/floribunda.v5i2.2015.131

Haerida I (2017) Liverworts of Bali, Indonesia, with new records to the island. Gardens' Bulletin (Singapore) 69(1): 81-87. https://doi.org/10.26492/gbs69(1).2017-05

Haerida I, Gradstein SR, Tjitrosoedirdjo SS (2010) Lejeuneaceae subfamily Ptychanthoideae (Hepaticae) in West Java. Gardens' Bulletin (Singapore) 62(1): 59-111.

Haerida I, Retnowati A, Djarwaningsih T, Suharna N, Ismail J (2015) Inventarisasi Keanekaragaman dan Potensi Flora, Jamur dan Lumut di Bali. Lembaga Ilmu Pengetahuan Indonesia (LIPI), Jakarta, 17 pp.

Haerida I, Rosalina D, Robiah Y (2020) Short notes on liverworts of Sumba, Lesser Sunda Islands, Indonesia. Hikobia 18: 97-98.

Hattori S (1951) On a small collection of Hepaticae from Dutch New Guinea. Botanical Magazine Tokyo 64(755/756): 112-119. https://doi.org/10.15281/jplantres 1887.64 .112 
Hattori S (1966) Anthocerotae and Hepaticae. In: Hara H (Ed.) The flora of Eastern Himalaya. Results of the botanical expedition to eastern Himalaya organized by the University of Tokyo 1960 and 1963. Univ. Tokyo Pr., Tokyo, 501-536.

Hattori S (1969) Studies of the Asiatic species of the genus Porella (Hepaticae). II. The Journal of the Hattori Botanical Laboratory 32: 319-359.

Hattori S (1975) Notes on the Asiatic species of the genus Frullania, Hepaticae. VII. The Journal of the Hattori Botanical Laboratory 39: 277-313.

Hattori S (1976) Studies of the Asiatic species of the genus Porella (Hepaticae). VI. The Journal of the Hattori Botanical Laboratory 40: 121-138.

Hattori S (1980) A revision of the subgenus Homotropantha of the genus Frullania, Hepaticae. The Journal of the Hattori Botanical Laboratory 47: 165-236.

Hattori S (1986) A synopsis of New Caledonian Frullaniaceae. The Journal of the Hattori Botanical Laboratory 60: 203-237.

Hegewald E, van Zanten BO (1986) A list of bryophytes from Bali (Indonesia) collected by E. and P. Hegewald in 1981. The Journal of the Hattori Botanical Laboratory 60: 263-269.

Heinrichs J, Dong S, Feldberg K, Schäfer-Verwimp A, Schmidt AR (2012) Sphaerolejeunea (Lejeuneaceae, Porellales) is a synonym of Lejeunea. Phytotaxa 69(1): 7-15. https://doi. org/10.11646/phytotaxa.69.1.3

Heinrichs J, Dong S, Schäfer-Verwimp A, Peralta DF, Feldberg K, Schmidt AR, Schneider H (2014a) Towards a monophyletic classification of Lejeuneaceae II: Subtribes Pycnolejeuneinae and Xylolejeuneinae subtr. nov, transfer of Otolejeunea to Lepidolejeuneinae, and generic refinements. Phytotaxa 163(2): 61-76. https://doi.org/10.11646/phytotaxa.163.2.1

Heinrichs J, Schäfer-Verwimp A, Czumay A, Dong S, Scheben A, Feldberg K, Schneider H (2014b) Towards a monophyletic classification of Lejeuneaceae I: Subtribe Leptolejeuneinae subtr. nov. Phytotaxa 156(3): 165-174. https://doi.org/10.11646/phytotaxa.156.3.7

Herzog T (1934) Studien über Drepanolejeunea II. Annales Bryologici 7: 57-94.

Inoue H (1984) The genus Plagiochila (Dum.) Dum. in southeast Asia. Academia Scientific Books, Tokyo, 142 pp.

Inoue H (1989) The bryophytes of Sabah (north Borneo) with special reference to the BRYOTROP transect of Mount Kinabalu V. Plagiochila (Plagiochilaceae, Hepaticae). Willdenowia 18(2): 555-567.

Jepson P, Whittaker RJ (2002) Ecoregions in context: A critique with special reference to Indonesia. Conservation Biology 16(1): 42-57. https://doi.org/10.1046/j.15231739.2002.01143.x

Jovet-Ast S (1967) Compléments à l'étude des Colura: Localités nouvelles; description d'une espèce nouvelle de Borneo. Revue Bryologique et Lichénologique 35(1/4): 143-148.

Jovet-Ast S (2000) Documents pour la connaissance des Riccia australiens (Hépatiques, Marchantiales) - nouvelles récoltes. Taxons nouveaux. Commentaires morphologiques et écologiques. Cryptogamie. Bryologie 21(4): 289-343. https://doi.org/10.1016/S12900796(00)01042-7

Jovet-Ast S (2003) Riccia des sous-genres Riccia et Ricciella récoltées en Indie et en Indonésie. Cryptogamie. Bryologie 24(3): 209-228. 
Jovet-Ast S, Schmid M (1958) Bryophytes du Haut-Donaï et du Darlac (Viet-Nam). Revue Bryologique et Lichénologique 27(3/4): 195-200.

Juslén A (2006) Revision of Asian Herbertus (Herbertaceae, Marchantiophyta). Annales Botanici Fennici 43(6): 409-436.

Kamimura M (1975) A small collection of liverworts from Java and Borneo II. Bulletin of Kochi Gakuen Junior College 6: 5-15.

Kitagawa N (1981) Miscellaneous notes on little-known species of Hepaticae, 51-70. Hikobia (suppl. 1): 67-72.

Krayesky DM, Crandall-Stotler BJ, Stotler RE (2005) A revision of the genus Fossombronia Raddi in the east Asia and Oceania. The Journal of the Hattori Botanical Laboratory 98: 1-45.

Kusuma AJ (2017) Pengaruh Norma HAM Terhadap Proses Kemerdekaan Timor Leste dari Indonesia. Otoritas. Jurnal Ilmu Pemerintahan 7(1): 1-13. https://doi.org/10.26618/ojip.v7i1.420

Ligrone R, Duckett JG, Renzaglia KS (2012) Major transitions in the evolution of early land plants: A bryological perspective. Annals of Botany 109(5): 851-871. https://doi. org/10.1093/aob/mcs017

Long DG (2006) Revision of the genus Asterella P. Beauv. in Eurasia. Bryophytorum Bibliotheca 63: 1-299.

Long DG, Grolle R (1990) Hepaticae of Bhutan II. The Journal of the Hattori Botanical Laboratory 68: 381-440.

McNeill J (2014) Holotype specimens and type citations: General issues. Taxon 63(5): 11121113. https://doi.org/10.12705/635.7

Miller HA (1968) Hepaticae from Truk, Caroline Islands. Micronesica 4: 239-254.

Miller HA, Whittier HO, Whittier BA (1983) Prodromus florae hepaticarum Polynesiae. Catalogue of Hepaticae and Anthocerotae. Bryophytorum Bibliotheca 25: 1-423.

Mitten W (1885) The Southeastern Moluccas. Musci et Hepaticae. In: Hemsley W (Ed.) Report on the Scientific Results of the voyage of H. M. S. Challenger. Botany. Vol. 1. III. part 2. Taylor et A.D. Poyser Limited, London, 212-214.

Mizutani M (1967) Studies of the Himalayan species of Bazzania. The Journal of the Hattori Botanical Laboratory 30: 71-90.

Monk KA, De Freter V, Reksodihardjo LG (1997) The ecology of Nusa Tenggara and Maluku (The ecology of Indonesia Series Volume V). Periplus Edition, Singapore.

Patzak SDF, Renner MAM, Schäfer-Verwimp A, Feldberg K, Heslewood MM, Fernandez Peralta D, Matos de Souza A, Schneider H (2016) A phylogeny of Lophocoleaceae-Plagiochilaceae-Brevianthaceae and a revised classification of Plagiochilaceae. Organisms, Diversity \& Evolution 16(3): 481-495. https://doi.org/10.1007/s13127-015-0258-y

Piippo S (1985) Bryophyte flora of the Huon Peninsula, Papua New Guinea. XII. Geocalycaceae (Hepaticae). Acta Botanica Fennica 131: 129-167.

Piippo S (1989a) Bryophyte flora of the Huon Peninsula, Papua New Guinea. XXX. Plagiochilaceae (Hepaticae). Annales Botanici Fennici 26(2): 183-236.

Piippo S (1989b) The bryophytes of Sabah (North Borneo) with special reference to the BRYOTROP transect of Mount Kinabalu. III. Geocalycaceae. Willdenowia 18(2): 513-527.

Piippo S (1993) On the taxonomy and nomenclature of SW Asiatic Geocalycaceae (Hepaticae). Annales Botanici Fennici 30(3): 195-203. 
Piippo S, Tan BC (1992) Novelties for the Philippine hepatic flora. The Journal of the Hattori Botanical Laboratory 72: 117-126.

Pócs T (1971) [An investigation of the mountain bryoflora of the Hashang province (Democratic Republic of Vietnam). Hepaticae]. Botanicheskii Zhurnal 56(5): 670-677.

Pócs T (1980) The epiphytic biomass and its effect on the water balance of two rain forest types in the Uluguru Mountains (Tanzania, East Africa). Acta Botanica Academiae Scientiarum Hungaricae, Budapest 26: 143-167.

Pócs T, Chantanaorrapint S (2016) Southern Thailand Bryophytes III: A preliminary study on non-epiphyllous taxa in lowland areas. Songklanakarin Journal of Science and Technology 38(3): 311-318.

Pócs T, Tixier P, Jovet-Ast S (1967) Seconde contribution à la bryoflore du Nord Vietnam. Botanikai Közlemények 54(1): 27-38. [Adatok Észak-Vietnám mohaflórájához. II]

Pócs T, Sass-Gyarmati A, Naikatini A, Tuiwawa M, Braggins JE, Pócs S, von Konrat MJ (2011) New liverwort (Marchantiophyta) records for the Fiji Islands. Telopea 13(3): 455-494. https://doi.org/10.7751/telopea20116031

Reilly SB (2016) Historical Biogeography of Reptiles and Amphibians from the Lesser Sunda Islands of Indonesia. University of California, Berkeley, ProQuest Dissertations Publishing. [10251004]

Renner MAM (2021) The typification of Australasian Plagiochila species (Plagiochilaceae: Jungermanniidae): a review with recommendations. New Zealand Journal of Botany: 1-53. https://doi.org/10.1080/0028825X.2020.1859557

Renner MAM, Heslewood MM, Patzak SD, Schäfer-Verwimp A, Heinrichs J (2016) The genera Chiastocaulon, Cryptoplagiochila and Pedinophyllum (Plagiochilaceae) in Australia. Australian Systematic Botany 29(4/5): 358-402. https://doi.org/10.1071/SB16029

Renner MAM, Patzak SDF, Heslewood MM, Schäfer-Verwimp A, Heinrichs J (2017) Third time lucky? Another substantially revised sectional classification for Australasian Plagiochila (Plagiochilaceae. Australian Systematic Botany 30(1): 70-104. https://doi.org/10.1071/ SB16038

Retnowati A, Rugayah JSR, Arifiani D (2019) Status Keanekaragaman Hayati Indonesia: Kekayaan Jenis Tumbuhan dan Jamur Indonesia. LIPI Press, Jakarta, 139 pp.

Rosyanti, Afriyansyah B, Haerida I (2018) Keanekaragaman Lumut di Kebun Botani Bangka Flora Society, Bangka. Floribunda 5(8): 315-321. [Diversity of bryophytes from botanical garden of Bangka Flora Society, Bangka]

Sande Lacoste CM (1856) Synopsis hepaticarum javanicarum. C. G. van der Post, Amsterdam, $112 \mathrm{pp}$.

Sande Lacoste CM (1864) Hepaticae. Jungermanniae archipelagi indici. Annales Musei Botanici Lugduno-Batavi 1: 287-314.

Schäfer-Verwimp A (2006) A new species of Diplasiolejeunea (Lejeuneaceae, Jungermanniopsida) from Sumatra, and a key for the genus in Asia. Herzogia 19: 239-244.

Schäfer-Verwimp A (2009) New national and regional bryophyte records, 22. Journal of Bryology 31(3): 201-210. https://doi.org/10.1179/037366809X12469790518367

Schäfer-Verwimp A, Feldberg K, Dong S, van Melick H, Peralta DF, Schmidt AR, Schneider H, Heinrichs J (2014) Towards a monophyletic classification of Lejeuneaceae III: The sys- 
tematic position of Leiolejeunea. Phytotaxa 170(3): 187-198. https://doi.org/10.11646/ phytotaxa.170.3.4

Schiffner V (1898) Conspectus hepaticarum archipelagi indici. Staatsdruckerei, Batavia, 382 pp. Schiffner V (1900) Die Hepaticae der Flora von Buitenzorg. I. Band. E. J. Brill, Leiden, 220 pp. Schiffner V (1955) Die Lebermoose der Deutschen Limnologischen Sunda-Expedition. Archiv für Hydrobiologie (suppl. 21)(3/4): 382-407.

Sharma D, Srivastava SC (1993) Indian Lepidoziinae (a taxonomic revision). Bryophytorum Bibliotheca 47: 1-353.

Shu L, Zhu R-L (2019) Leptolejeunea nigra (Lejeuneaceae), a new species with brownish black ocelli based upon morphology and DNA. Phytotaxa 427(1): 31-42. https://doi. org/10.11646/phytotaxa.427.1.4

Siregar ES (2015) The liverworts (Marchantiophyta) of Mount Sibayak North Sumatra [Lumut hati (Marchantiophyta) di Gunung Sibayak Sumatra Utara]. Bogor Agricultural University, Bogor, Java, 105 pp. http://repository.ipb.ac.id/handle/123456789/77268

Siregar ES, Pasaribu N (2020) The liverwort family Lejeuneaceae (Marchantiophyta) of Mount Lubuk Raya, North Sumatra, Indonesia. Biodiversitas (Surakarta) 21(6): 2767-2776. https://doi.org/10.13057/biodiv/d210653

Siregar ES, Ariyanti NS, Tjitrosoedirdjo SS (2013) The liverwort genus Marchantia (Marchantiaceae) of Mount Sibayak, North Sumatra, Indonesia. Biotropia 20(2): 73-80. https://doi. org/10.11598/btb.2013.20.2.327

Siregar ES, Ariyanti NS, Tjitrosoedirdjo SS (2014) Lejeuneaceae anak suku Ptychanthoideae di Hutan Sibayak, Sumatra Utara. Floribunda 4(8): 218-225. [Lejeuneaceae subfamily Ptychanthoideae in Sibayak Forest, North Sumatra] https://doi.org/10.32556/floribunda.v4i8.2014.118

Siregar ES, Hannum S, Pasaribu N (2017) Lejeuneaceae (Marchantiophyta) of Sicike-cike Natural Park, North Sumatra Indonesia. Taiwania 62(4): 356-362. https://doi.org/10.6165/ tai.2017.62.356

Siregar ES, Ariyanti NS, Tjitrosoedirdjo SS (2018) Plagiochila (Marchantiophyta) of Mount Sibayak, North Sumatra. IOP Conference Series: Earth and Environmental Science 130 (012017): 1-11. https://doi.org/10.1088/1755-1315/130/1/012017

So ML (2001) Plagiochila (Hepaticae, Plagiochilaceae) in China. Monographs in Systematic Botany 60: 1-214. https://doi.org/10.2307/25027887

So ML (2003a) The genus Schistochila in Asia. The Journal of the Hattori Botanical Laboratory 93: 79-100.

So ML (2003b) The genus Metzgeria (Hepaticae) in Asia. The Journal of the Hattori Botanical Laboratory 94: 159-177.

So ML, Grolle R (1999) Studies on Plagiochila in Asia: Supplements to sections Abietinae, Annotinae, Ciliatae, Contiguae, Cucullatae, Poeltiae, Subtropicae and Zonatae. Cryptogamie. Bryologie 20(3): 167-179. https://doi.org/10.1016/S1290-0796(99)80014-5

Söderström L, Séneca A (2008) Can species richness and richness of range restricted species be used as Tool for Conservation. In: Mohamed H, Baki BB, Nasrulhaq-Boye A, Lee PKY (Eds) Bryology in the New Millennium, 439-450.

Söderström L, Gradstein SR, Hagborg A (2010) Checklist of the hornworts and liverworts of Java. Phytotaxa 9: 53-149. https://doi.org/10.11646/phytotaxa.9.1.7 
Söderström L, Hagborg A, von Konrat M, Renner MAM (2008) Chapter Ten: Early Land Plants Today: Liverwort checklist of checklists. Fieldiana Botany 47: 105-130. https://doi. org/10.3158/0015-0746-47.1.105

Söderström L, Hagborg A, von Konrat M, Bartholomew-Began S, Bell D, Briscoe L, Brown E, Cargill DC, Costa DP, Crandall-Stotler BJ, Cooper E, Dauphin G, Engel J, Feldberg K, Glenny D, Gradstein SR, He X, Hentschel J, Ilkiu-Borges AL, Katagiri T, Konstantinova NA, Larraín J, Long D, Nebel M, Pócs T, Puche F, Reiner-Drehwald E, Renner M, SassGyarmati A, Schäfer-Verwimp A, Segarra-Moragues J, Stotler RE, Sukkharak P, Thiers B, Uribe J, Váňa J, Wigginton M, Zhang L, Zhu R-L (2016) World checklist of hornworts and liverworts. PhytoKeys 59: 1-828. https://doi.org/10.3897/phytokeys.59.6261

Srivastava SC, Sharma D (2000) A preliminary study on the liverwort and hornwort flora of Silent Valley (Kerala). In: Chauhan DK (Ed.) Prof. D.D. Nautiyal Commemoration Volume: Recent Trends in Botanical Researches. University of Allahabad, Allahabad, 55-75.

Srivastava A, Srivastava SC (2002) Indian Geocalycaceae (Hepaticae). A taxonomic study. Bishen Singh Mahendra Pal Singh, Dehra Dun, 246 pp.

Stephani F (1886) Hepaticarum species novae vel minus cognitae VIII. Hedwigia 25(6): 233-249. Stephani F (1899) Species hepaticarum 1. Bulletin de l'Herbier Boissier 7(7): 518-533. https:// doi.org/10.5962/bhl.title.95494

Stephani F (1907) Species hepaticarum 3. Bulletin de l'Herbier Boissier (sér. 2) 7(10): 837-852. https://doi.org/10.5962/bhl.title.95494

Stephani F (1908) Species hepaticarum 3. Bulletin de l'Herbier Boissier (sér. 2) 8(10): 745-776. https://doi.org/10.5962/bhl.title.95494

Stephani F (1909) Species hepaticarum 3. George \& Cie, Genève \& Bale, 517-693. https:// doi.org/10.5962/bhl.title.95494

Stephani F (1911) Species hepaticarum 4. George \& Cie, Genève \& Bale, 449-736. https:// doi.org/10.5962/bhl.title.95494

Stephani F (1917) Species hepaticarum 6. George \& Cie, Genève \& Bale, 1-128. https://doi. org/10.5962/bhl.title.95494

Stephani F (1924) Species hepaticarum 6. George \& Cie, Genève \& Bale, 433-622.

Sukkharak P (2011) Taxonomy and phylogeny of the liverwort genus Thysananthus (Marchantiophyta: Lejeuneaceae). Georg-August Universität, Göttingen, 189 pp.

Sukkharak P (2015) A systematic monograph of the genus Thysananthus (Lejeuneaceae, Marchantiophyta). Phytotaxa 193(1): 1-81. https://doi.org/10.11646/phytotaxa.193.1.1

Sukkharak P, Gradstein SR (2014) A taxonomic revision of the genus Mastigolejeunea (Marchantiophyta: Lejeuneaceae). Nova Hedwigia 99(3/4): 279-345. https://doi. org/10.1127/0029-5035/2014/0206

Swanson ES, Miller HA (1969) Hepaticae from Palau, Caroline Islands, II. Enumeration. Micronesica 5: 139-149.

Thiers BM (1993) A monograph of Pleurozia (Hepaticae, Pleuroziaceae). The Bryologist 96(4): 517-554. https://doi.org/10.2307/3243984

Tixier P (1962) Bryophytes du Vietnam. Premières récoltes dans le massif de Bach-Ma. Revue Bryologique et Lichénologique 31(3/4): 190-203. 
Tixier P (1973) Bryophytae Indosinicae. Liverworts collected in Thailand. Natural History Bulletin of the Siam Society 24(3/4): 449-456.

Tixier P (1974) Bryophytes exotiques: Bryophytes des Nouvelle-Hebrides (Expedition de la Royal Society). Bulletin du Muséum National d'Histoire Naturelle (sér. 3). Botanique 16(269): 33-45.

Tixier P (1979) Bryogéographie du Mont Bokor (Cambodge) (Bryophyta indosinica XXIV). Bryophytorum Bibliotheca 18: 5-121.

Touw A (1992) A Survey of The Mosses of the Lesser Sunda Islands (Nusa Tenggara), Indonesia. The Journal of the Hattori Botanical Laboratory 71: 289-366.

van Steenis-Kruseman MJ, van Steenis CGGJ (1950) Malaysian plant collectors and collections being a Cyclopaedia of botanical exploration in Malaysia and a guide to the concerned literature up to the year 1950. Flora Malesiana - Series 1. Spermatophyta 1(1): 2-639.

van Welzen PC, Slik JWF, Alahuhta J (2005) Plant distribution patterns and plate tectonics in Malesia. In: Friis I, Balslev H (Eds) Proceedings of a symposium on plant diversity and complexity patterns: local, regional and global dimensions. Biologiske Skrifter, The Royal Danish Academy of Sciences and Letters, Copenhagen, 199-217.

Váňa J (1972) Miscellaneous notes on the Asiatic Jungermannioideae II. Journal of the Hattori Botanical Laboratory 36: 57-74.

Váňa J (1973) Lebermoose aus Neuguinea. 10. Jungermannia. The Journal of the Hattori Botanical Laboratory 37: 185-190.

Váňa J (1975) Studien über die Jungermannioideae (Hepaticae). 8. Jungermannia subg. Plectocolea und Solenostoma in Australien, Neuseeland und Ozeanien. Folia Geobotanica et Phytotaxonomica 10(3): 277-323. https://doi.org/10.1007/BF02854717

Váňa J (1991a) The bryophytes of Sabah (north Borneo) with special reference to the BRYOTROP transect of Mt. Kinabalu. XIII. Jungermanniaceae (Hepaticopsida, Jungermanniales). Willdenowia 20: 171-183.

Váňa J (1991b) The bryophytes of Sabah (North Borneo) with special reference to the BRYOTROP transect of Mt. Kinabalu. XV. Lophoziaceae. Willdenowia 20: 199-219.

Váňa J, Piippo S (1989a) Bryophyte flora of the Huon Peninsula, Papua New Guinea. XXIX. Jungermanniaceae and Gymnomitriaceae (Hepaticae). Annales Botanici Fennici 26(2): $107-125$.

Váňa J, Piippo S (1989b) Bryophyte flora of the Huon Peninsula, Papua New Guinea. XXXI. Cephaloziaceae subfam. Alobielloideae, Cephaloziellaceae, Antheliaceae and Lophoziaceae (Hepaticae). Annales Botanici Fennici 26(3): 263-290.

Váňa J, Ochyra R, Lebouvier M, Cykowska-Marzencka B (2014) Bryophytes of Île Amsterdam in the South Indian Ocean: 1. Liverworts. Cryptogamie. Bryologie 35(4): 335-371. https://doi.org/10.7872/cryb.v35.iss4.2014.335

Verdoorn F (1930) Die Frullaniaceae der Indomalesischen Inseln (De Frullaniaceis VII). Annales Bryologici (suppl. 1): 1-187. https://doi.org/10.1007/978-94-015-5385-8

Verdoorn F (1934a) De Frullaniaceis XV. Die Lejeuneaceae Holostipae der Indomalaya unter Berücksichtung sämtlicher aus Asien, Australien, Neuseeland und Ozeanien angeführten Arten. Annales Bryologici (suppl. 4): 40-192. https://doi.org/10.1007/978-94-015-5442-8_2 
Verdoorn F (1934b) De Frullaniaceis XVII. Über neue Frullania-Sammlungen und die Verbreitung der Jubuleae. Annales Bryologici (suppl. 4): 203-224. https://doi.org/10.1007/97894-015-5442-8_4

Verdoorn F (1935) Hepaticae selectae et criticae, series VII (1934) et series VIII (1935) and Musci selecti et critici, series I (1934) et series II (1935). Annales Bryologici 8: 150-158.

Verdoorn F (1937) On some new collections of Asiatic and Oceanic Jubulae (De Frullaniaceis XVIII). Blumea (suppl. 1): 210-213.

von Konrat M, Söderström L, Hagborg A (2010) The Early Land Plants Today project: A community-driven effort and a new partnership with Phytotaxa. Phytotaxa 9(1): 11-21. https://doi.org/10.11646/phytotaxa.9.1.4

Wilson R, Gradstein SR, Schneider H, Heinrichs J (2007) Unravelling the phylogeny of Lejeuneaceae (Jungermanniopsida): Evidence for four main lineages. Molecular Phylogenetics and Evolution 43(1): 270-282. https://doi.org/10.1016/j.ympev.2006.10.017

Wilson R, Gradstein SR, Heinrichs J, Groth H, Ilkiu-Borges AL, Hartmann FA (2004) Phylogeny of Lejeuneaceae: a cladistic analysis of chloroplast gene rbcL sequences and morphology with preliminary comments on the mitochondrial NAD4-2 spacer region. Monographs in Systematic Botany from the Missouri Botanical Garden 98: 189-202.

Winter G, Schäfer-Verwimp A (2020) Re-evaluation of the taxonomic status of Frullania caulisequa and Frullania obcordata (Frullaniaceae, Marchantiophyta). Frahmia 19: 1-21.

Yamada K, Hayashi M (2003) Studies on the bryophyte flora of Vanuatu. 8. Heteroscyphus (Geocalycaceae, Hepaticae). Bulletin of the National Science Museum, Tokyo Series B Botany 29: 149-152.

Ye W, Zhu RL (2018) Gaolejeunea, a new genus from China and new member of subtribe Echinolejeuneinae (Lejeuneaceae, Marchantiophyta). The Bryologist 121(1): 41-48. https:// doi.org/10.1639/0007-2745-121.1.041

Zhang J, Fu XX, Li RQ, Zhao X, Liu Y, Li M-H, Zwaenepoel A, Ma H, Goffinet B, Guan Y-L, Xue J-Y, Liao Y-Y, Wang Q-F, Wang Q-H, Wang J-Y, Zhang G-Q, Wang Z-W, Jia Y, Wang M-Z, Dong S-S, Yang J-F, Jiao Y-N, Guo Y-L, Kong H-Z, Lu A-M, Yang H-M, Zhang S-Z, de Peer YV, Liu Z-J, Chen Z-D (2020) The hornwort genome and early land plant evolution. Nature Plants 6(2): 107-118. https://doi.org/10.1038/s41477-019-0588-4

Zhu R-L, Gradstein SR (2005) Monograph of Lopholejeunea (Spruce) Schiffn. (Lejeuneaceae, Hepaticae) in Asia. Monographs in Systematic Botany from the Missouri Botanical Garden 74: 1-95.

Zollinger H (1854) Systematisches Verzeichniss der im indischen Archipel in den Jahren 1842 1848 gesammelten sowie der aus Japan empfangenen Pflanzen. 1 Heft, Zürich, Kiesling, 1-80. https://doi.org/10.5962/bhl.title.53656 